\title{
Spectrum Sharing in an ISM Band: Outage Performance of a Hybrid DS/FH Spread Spectrum System with Beamforming
}

\author{
Hanyu Li, Mubashir Syed, Yu-Dong Yao, and Theodoros Kamakaris \\ Wireless Information Systems Engineering Laboratory (WISELAB), Department of Electrical \& Computer Engineering, \\ Stevens Institute of Technology, Hoboken, NJ 07030, USA \\ Correspondence should be addressed to Yu-Dong Yao, yyao@stevens.edu
}

Received 15 February 2009; Revised 19 May 2009; Accepted 16 September 2009

Recommended by R. Chandramouli

\begin{abstract}
This paper investigates spectrum sharing issues in the unlicensed industrial, scientific, and medical (ISM) bands. It presents a radio frequency measurement setup and measurement results in $2.4 \mathrm{GHz}$. It then develops an analytical model to characterize the coexistence interference in the ISM bands, based on radio frequency measurement results in the $2.4 \mathrm{GHz}$. Outage performance using the interference model is examined for a hybrid direct-sequence frequency-hopping spread spectrum system. The utilization of beamforming techniques in the system is also investigated, and a simplified beamforming model is proposed to analyze the system performance using beamforming. Numerical results show that beamforming significantly improves the system outage performance. The work presented in this paper provides a quantitative evaluation of signal outages in a spectrum sharing environment. It can be used as a tool in the development process for future dynamic spectrum access models as well as engineering designs for applications in unlicensed bands.
\end{abstract}

Copyright (C) 2009 Hanyu Li et al. This is an open access article distributed under the Creative Commons Attribution License, which permits unrestricted use, distribution, and reproduction in any medium, provided the original work is properly cited.

\section{Introduction}

Radio frequency (RF) has become one of the most precious resources with the booming usage of wireless applications in the recent years. Licensing radio frequencies for commercial use has long been the mechanism adopted by regulatory bodies for managing the RF spectrum. An exclusive license was granted to protect the licensee's service from interference, but it also excluded shared use even when the licensee is absent. Part of the reason for this approach was the original technological limitations. Although technology has evolved over time and overcome most of these limitations, regulatory spectrum management methodology has not been changed. On the other hand, the industrial, scientific, and medical (ISM) radio bands, originally allocated for noncommercial uses, were later modified to allow for more services [1], prompting an influx of wireless communication applications. Those applications, including wireless local area networks (WLANs) and Bluetooth, take advantage of these bands for license-free operation. This can be seen as an indication of the role that unlicensed bands are set to play in the evolution of wireless communications towards spectrum sharing or dynamic spectrum access.

Efficient coexistence technology is essential for successful operation of systems in the unlicensed band since there is no protection from interference caused by coexisting systems. This requires a multifaceted approach to system design which includes spectrum occupancy measurements, modeling of coexistence interference, performance evaluation, and development of optimum waveforms. A number of studies of spectrum occupancy measurements in the ISM band have been reported in [2-5]. At Stevens Institute of Technology, an investigative study is being carried out for distributed spectrum occupancy measurements in the 2.4 GHz ISM band [6]. Based on measurement data in typical environments (indoor, outdoor, etc.), an analytical model of the coexistence interference was investigated in [7]. The model illustrates a simple approach to interference modeling due to uncoordinated sources/technologies, which share a common band of frequencies.

Beamforming, a multiple antenna technique, has received great attention in wireless communications recently 


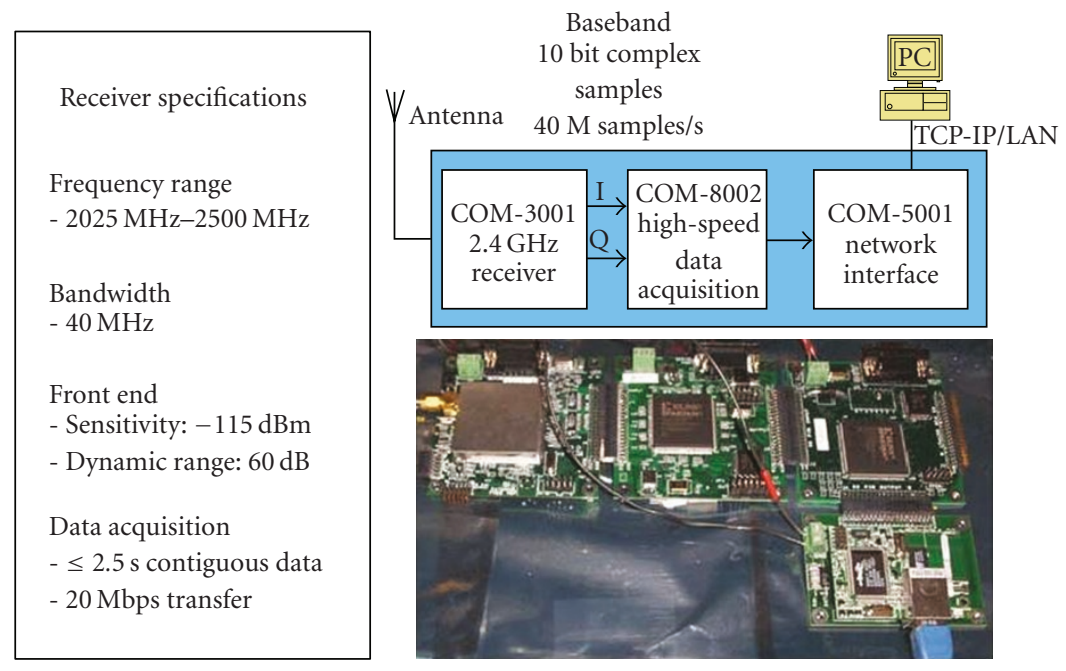

FIGURE 1: Measurement setup.

as it provides solutions to problems such as increasing interference, limited bandwidth, and limited transmission range [8]. In an interference rich scenario such as ISM band, beamforming is expected to play an important role. Beamforming uses arrays of antennas to control the RF radiation pattern. When receiving a signal, beamforming can increase the gain in the direction of desired signal and decrease the gains in the directions of interferences. When transmitting a signal, beamforming can increase the gain in the direction of the signal. A preliminary study of a single hybrid direct-sequence and frequency-hopping (DS/FH) signal operating in an ISM band was presented in [7], in which beamforming was not considered. This paper investigates a multiple user DS/FH system using beamforming.

The rest of the paper is structured as follows. In the next section, we first present an RF measurement setup and measurement results in the $2.4 \mathrm{GHz}$ ISM band. We then discuss the $2.4 \mathrm{GHz}$ ISM band occupancy scenarios and describe our approach to characterize interference for a typical application environment. The signal model that is considered for the analysis in this paper is explained in the subsequent section. Section 4 presents mathematical derivations of expressions for outage probabilities. Error performance analysis is discussed in Section 5. Numerical results are presented in Section 6. Finally, the conclusions are drawn in Section 7.

\section{Interference Measurement and Modeling}

2.1. Measurement Setup. In order to develop a system capable of distributed spectrum measurements, 10-20 inexpensive, portable, off-the-shelf, lightweight and Ethernet interfaced measurement devices are used. ComBlocks [9] are small commercial off-the-shelf modules which are preprogrammed with essential communication processing functions, including modulation, demodulation, error correction encoding and decoding, digital to analog/RF, RF/analog to digital, formatting, data storage, and baseband interface. With two or more ComBlock modules interfaced with each other, we build the whole data measurement system based on our requirements.

Figure 1 illustrates a ComBlock receiver assembly for the 2.4 GHz ISM band with $40 \mathrm{MHz}$ bandwidth. The baseband signal is digitized and sent to a data acquisition server over LAN. The ComBlock assemblies are fully controlled over LAN by our MATLAB-based application that coordinates data acquisition from multiple distributed ComBlock assemblies and allows flexible signal processing. Using this configuration we can capture up to 2.5 seconds of continuous signal, or smaller segments for spectral analysis with a capture to processing ratio of $1 \%$. The RF front end has a frequency range from $2025 \mathrm{MHz}$ to $2500 \mathrm{MHz}$, sensitivity of $-115 \mathrm{dBm}$, and a dynamic range of $60 \mathrm{~dB}$.

2.2. Measurement Results and Modeling. Using the RF setup (Figure 1), we obtained measurement results and show in Figure 2 some sample data (spectrogram at microsecond $/ 10 \mathrm{KHz}$ resolution) observed in the $2.4 \mathrm{GHz}$ ISM band that highlight spectrum occupancy by typical devices. From the temporal and spectral emission characteristics, various applications have been identified, including Bluetooth, IEEE 802.11b WLAN, and microwave oven emissions. Additional tone and narrowband emissions have also been observed.

In view of the variations in bandwidth occupancy patterns of coexisting wireless devices and several other factors (such as proximity constraints and application environment), design considerations for effective introduction of additional signal transmissions in the ISM band necessitate that a threefold approach is employed. The first requirement is to minimize the interference to coexisting services. The second is to quantify the interference from coexisting users. Finally, building on the above two efforts, effective waveforms that are robust to interference need to be developed. Towards this end, we attempt to profile 


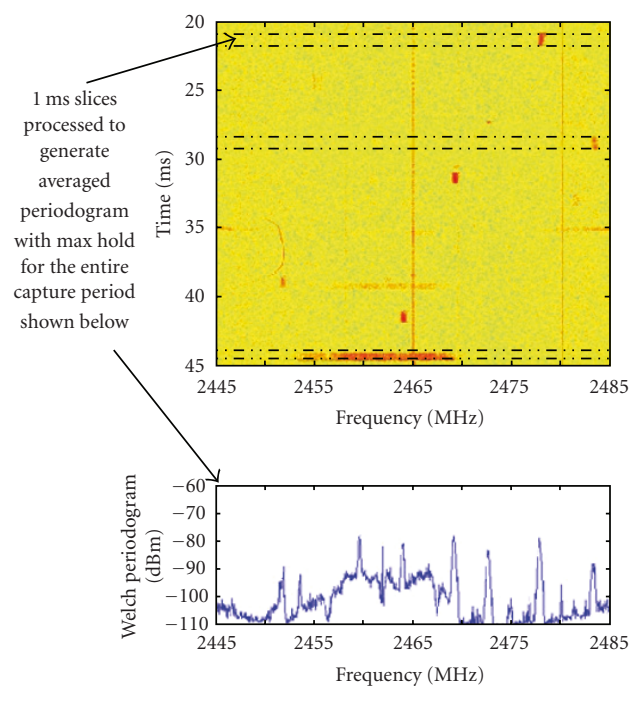

(a)

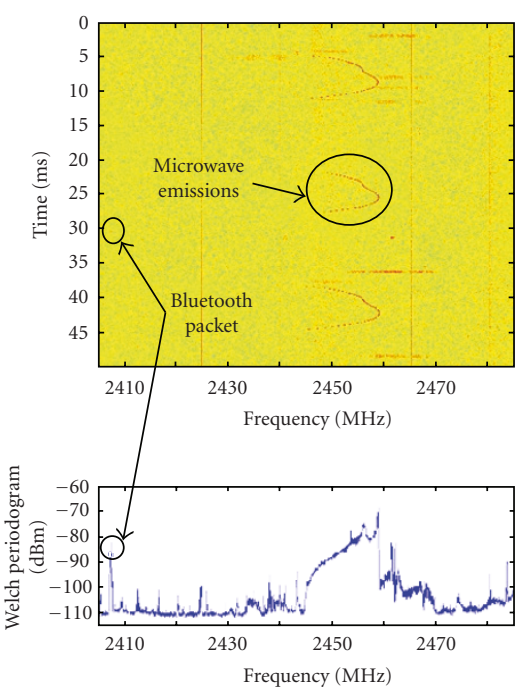

(b)
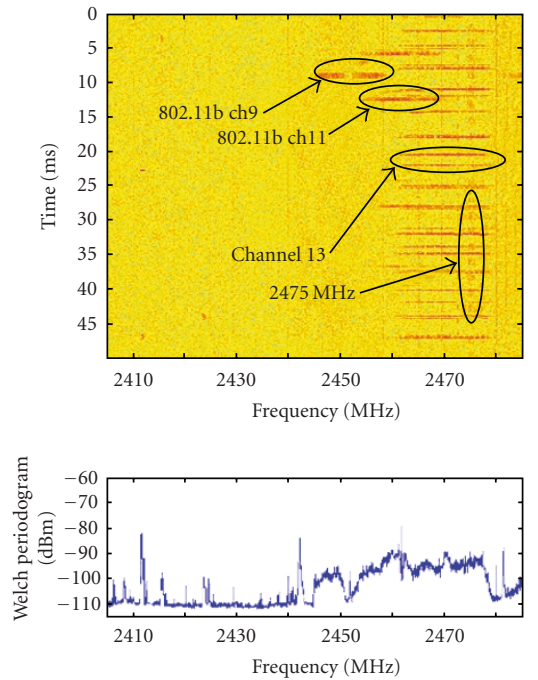

(c)

FIGURE 2: ISM band spectral emission measurement. (a) observations of Bluetooth packet; (b) observations of Bluetooth packets and microwave oven emissions; (c) observation of IEEE $802.11 \mathrm{~b}$ packets.

the observed emissions in terms of various representative interference types.

The spectral, spatial, and temporal characterizations of interferences are summarized in Table 1. It is noted that the profiling presented here is derived from the measurements conducted in a specific office environment with the usual set of devices currently typical in the $2.4 \mathrm{GHz}$ ISM band. The significance of this interference modeling approach is that it showcases a simple and sufficiently accurate methodology for profiling emissions in an unlicensed band that can be used for different interference scenarios. Assume that the bandwidth of the signal of interest is $B$ and the entire ISM band can be divided into $N$ frequency slots, each with bandwidth $B$. Transmissions from devices operating in ISM band can cover the entire signal bandwidth or a part of it. As listed in the table, the observed emissions are categorized into three broad interference types based on their transmission bandwidth-barrage, partial-band, and tone. Barrage type interferers are those whose transmission bandwidth covers the entire signal bandwidth $B$. Partialband interference is a generic grouping of interference sources that occupy part of the desired signal bandwidth $B$. Devices transmitting single frequency impulses are grouped under the tone interference type. To capture the effect of spatial characteristics of the different interference sources, emissions are also parameterized in terms of their received power levels as $Y_{i, j}$, where the first subscript corresponds to the interference type (i.e., $i \in\{1,2,3\}$ denoting barrage interference, partial-band interference, and tone interference resp.) and the second subscript, $j$, denotes a specific source of the given interference type. Emissions from different sources also have different temporal characteristics such as periodicity and duty cycle. Similar to power level, the duty cycle of each source is parameterized as $\rho_{i, j}$.

\section{Signal and Beamforming Models}

In order to introduce new signals in coexistence environment, an appropriate waveform has to be adopted. FH and DS have been widely used in ISM band. For example, Bluetooth uses FH [10] and Wi-Fi employs DS [11]. A hybrid DS/FH system has been implemented in [12] and analyzed in terms of spectral efficiency in [13]. Hybrid spread spectrum (SS), where a direct-sequence modulated signal is frequency hopped, is an attractive choice. DS/FH waveform has been used in fixed spectrum systems but it has great potential in dynamic spectrum access methodology since its inherent ability to dynamically change signal frequency and it can mitigate interference caused to others through $\mathrm{FH}$. Additional interference reduction is provided due to the DS spreading gain. It is noted that in keeping with a more generalized treatment of the approach presented in this paper and for lucidity of presentation, specifics have been avoided. For instance, explicit details of the signature sequence used for spreading, the frequency hopping pattern and the signal processing aspects of multipath fading have been ignored. Just enough detail is furnished so as to account for the concerned phenomena for our purposes.

3.1. Signal and Channel Model. For the analysis presented here, a DS/FH system with multiple users is considered and all the other transmitting sources occupying the frequency band are taken to be interferers. A binary phase shift keying (BPSK) modulation and asynchronous DS/FH system are considered. Let us denote the BPSK modulated DS/FH signal of user $i$ as $s_{i}(t)$, and is given by

$$
s_{i}(t)=\sqrt{2 X_{i}} c_{i}(t) b_{i}(t) \cos \left\{2 \pi\left[f_{c}+f_{i}(t)\right] t+\theta_{i}+\phi_{i}(t)\right\}
$$


TABLE 1: $2.4 \mathrm{GHz}$ ISM band interference characteristics based on measurements.

\begin{tabular}{|c|c|c|c|c|c|}
\hline Emissions & Interference type & Power level & Duty cycle & Periodicity & Bandwidth \\
\hline $802.11 \mathrm{~b}$ packets & Barrage & $Y_{1, j}$ & $\rho_{1, j}$ & No & $\gg B$ \\
\hline Microwave oven & Barrage & $Y_{1, j}$ & $\rho_{1, j}$ & Yes & $\gg B$ \\
\hline Bluetooth packets & Partial-band & $Y_{2, j}$ & $\rho_{2, j}$ & No & $\approx B$ \\
\hline Others & Tone & $Y_{3, j}$ & $\rho_{3, j}$ & Yes & $\ll B$ \\
\hline
\end{tabular}

where $f_{c}$ is the carrier frequency, $X_{i}$ is the power of the transmitted signal, and $\theta_{i}$ is the phase introduced by the BPSK modulator. The signal is frequency-hopped according to $f_{i}(t)$ and $\phi_{i}(t)$ is the phase waveform introduced by the frequency hopper. The data signal, $b_{i}(t)$, (which is a differentially encoded version of the information signal) is a sequence of rectangular pulses with amplitude equal to either +1 or -1 and its duration is $T$. The code waveform $c_{i}(t)$ is a periodic sequence of positive and negative rectangular pulses of unit amplitude and duration $T_{c}$. The processing gain (PG) of the system is defined as $G_{\mathrm{DS}}=T / T_{c}$.

We consider that the propagation channel for the desired signal is characterized by fading channel with impulse response $h_{i}(t)$. In a multipath environment, the impulse response $h_{i}(t)$ can be written as

$$
h_{i}(t)=\sum_{l=0}^{L-1} \beta_{i, l} e^{j \varphi_{i, l}} \delta\left(t-\tau_{i, l}\right)
$$

where $L$ is the number resolvable paths, $\beta_{i, l}$ is the amplitude, $\varphi_{i, l}$ is the phase shift, and $\tau_{i, l}$ is the delay. Assuming each path is following a Rayleigh fading, its power, $\gamma_{i, l}$, is following exponential distribution

$$
f_{\gamma_{i, l}}\left(\gamma_{i, l}\right)=\frac{1}{\Omega_{i, l}} \exp \left(-\frac{\gamma_{i, l}}{\Omega_{i, l}}\right), \quad \gamma_{i, l} \geq 0,
$$

where $\Omega_{i, l}=\mathrm{E}\left[\beta_{i, l}^{2}\right]$ is the average channel power. For fare comparison, the total power of multipath channel is normalized to one

$$
\sum_{l=0}^{L-1} \Omega_{i, l}=1
$$

For the wireless mobile channel, it has been found that the multipath intensity profile (MIP) usually follows the negative exponential relationship [14]

$$
\Omega_{i, l}=\Omega_{i, 0} e^{-l \delta}, \quad l=0,1, \ldots, L-1 .
$$

The decay factor $\delta$ reflects the decay rate of the average path strength as a function of the path delay. Thus, the signal at the input of the receiver is given by

$$
\begin{aligned}
r(t) & =\sum_{i=1}^{K} s_{i}(t) \otimes h_{i}(t)+y(t)+n(t) \\
& =\sum_{i=1}^{K} \sum_{l=0}^{L-1} \beta_{i, l} e^{j \psi_{i, l}} s_{i}\left(t-\tau_{i, l}\right)+y(t)+n(t),
\end{aligned}
$$

where $\otimes$ denotes the convolution operation, $y(t)$ denotes the total interference, $K$ is the number of users, and $n(t)$ is the additive white Gaussian noise (AWGN) with two-sided spectrum density $N_{0} / 2$. The receiver is assumed capable of acquiring the frequency-hopping pattern, signature sequence and time synchronization of the user. The output of the frequency dehopper in the receiver enters the despreader and then the BPSK demodulator.

3.2. Introduction of Simplified and Accurate Beamforming Model. Beamforming, a multiple antenna technique, is able to increase the gain in the direction of desired signal and decrease the gain in the direction of interference. A beamforming combining network connects an array of low gain antenna elements and could generate an antenna pattern [15]

$$
G(\psi, \theta)=\left|\frac{\sin (0.5 M \pi(\sin \theta-\sin \psi))}{M \sin (0.5 \pi(\sin \theta-\sin \psi))}\right|^{2},
$$

where $M$ is the number of antenna elements, $\theta$ is an arrival angle of incident waves, and $\psi$ is a scan angle. The beam could be steered to a desired direction by varying $\psi$. The complexity considering the exact beam pattern can be high, especially for performance evaluation under beamforming impairments such as DOA estimation errors, due to multiple integrals. A simple Bernoulli model is introduced in [16] in which a signal is considered to be within a mainlobe ( $G=1)$ or out of the mainlobe $(G=0)$ and the halfpower beamwidth is defined as the beamwidth. This model is easy to use, but it neglects the impact of sidelobes and the effect of any specific beam patterns. Reference [17] provides a beamforming model with a triangular pattern to characterize the mainlobe of a beam. In [18], a beamforming model having a flat mainlobe and a flat sidelobe is developed. The width of the mainlobe and the height of the sidelobe are calculated based on the first moment and the second moment of the real beam pattern. This model considers the impact of real beam pattern and it is proven to be accurate [19], but it is cumbersome to use if there are multiple types of interference since the derivation has to consider all the cases when a specific interferer is in the mainlobe or sidelobe. In this paper, we introduce a simple, yet accurate beamforming model and then use it to evaluate the performance of a Hybrid DS/FH spread spectrum system with beamforming. While evaluating the interference, there 


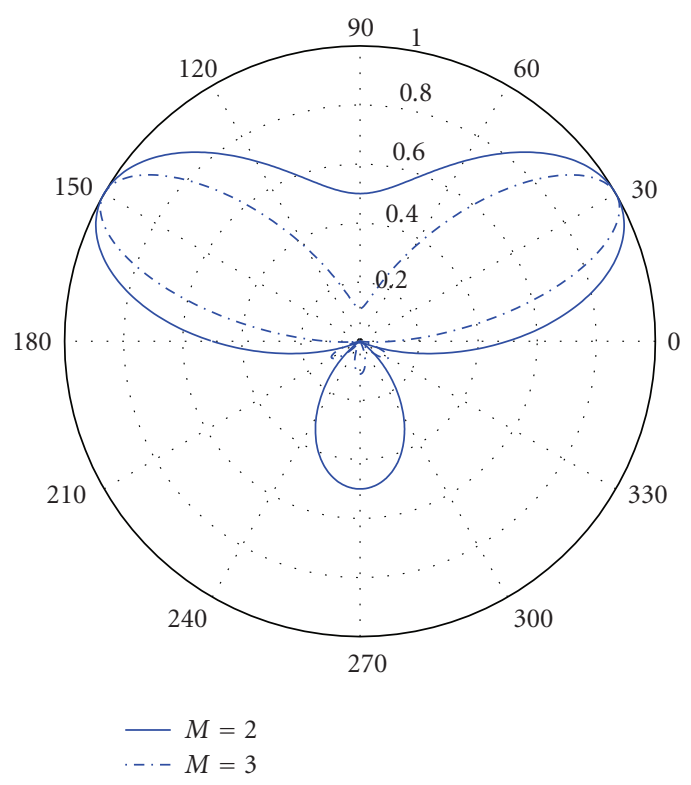

(a)

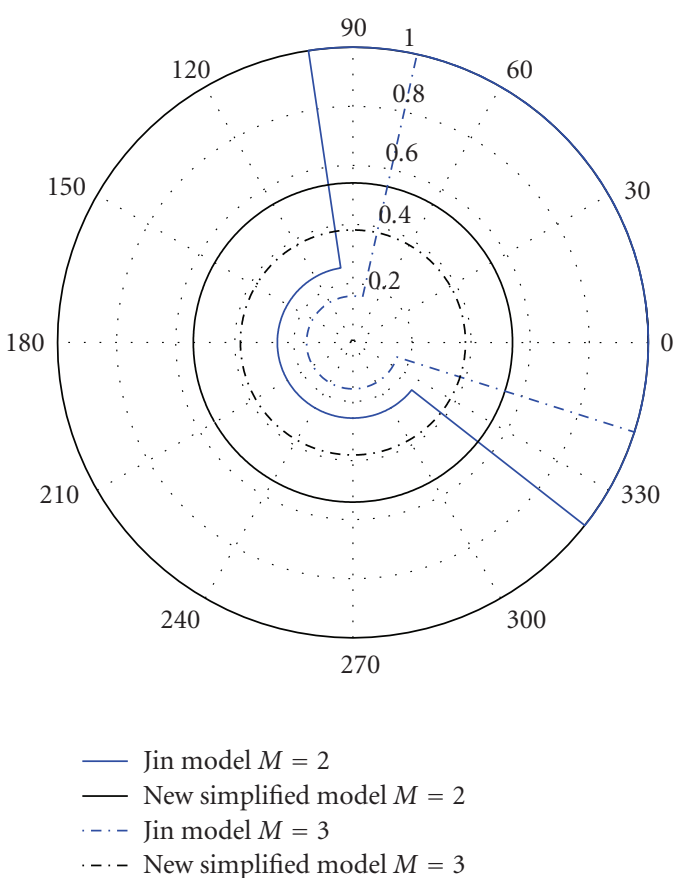

(b)

Figure 3: A simplified model for beamforming with arrival angle $\theta=30^{\circ}$. (a) Signal model, (b) interference model.

is only one parameter, $\alpha_{\mathrm{BF}}$, associated with the simplified model (Figure 3(b)). The parameter

$$
\begin{aligned}
\alpha_{\mathrm{BF}} & =\mathrm{E}[G(\psi, \theta)] \\
& =\iint_{0}^{2 \pi}\left|\frac{\sin (0.5 M \pi(\sin \theta-\sin \psi))}{M \sin (0.5 \pi(\sin \theta-\sin \psi))}\right|^{2} p_{\psi}(\psi) p_{\theta}(\theta) d \psi d \theta
\end{aligned}
$$

is the antenna gain averaged with respect to random variables, $\psi$ and $\theta$, in the region from 0 to $2 \pi$. While evaluating the signal, it uses the real beam pattern (Figure $3(\mathrm{a})$ ). In Figure 3, the model proposed in [18] is also plotted for comparison. It is noted that the simplified model differs with Jin's model in [18] on evaluating interference. Using the new simplified model to evaluate the performance of a wireless system with beamforming is simple. Just reduce all the interference power by $\alpha_{\mathrm{BF}}$ and all the existing results are still valid. For example, the outage probability of a wireless system in Rayleigh fading environment with $K$ interferers can be written as

$$
P_{\text {out }}=1-\frac{1}{\left(1+\left(\alpha_{\mathrm{BF}} / \mathrm{SIR}\right)\right)^{K}} .
$$

3.3. Accuracy of Simplified Model. We use (9) (an expression derived based on the simplified beamforming model) and results in [19] to calculate outage probabilities of wireless systems with beamforming. The numerical results are shown in Figure 4. It can be seen that the evaluation results using the simplified model match those using the actual beam pattern very well. For comparison, the Jin's model in [18] is also

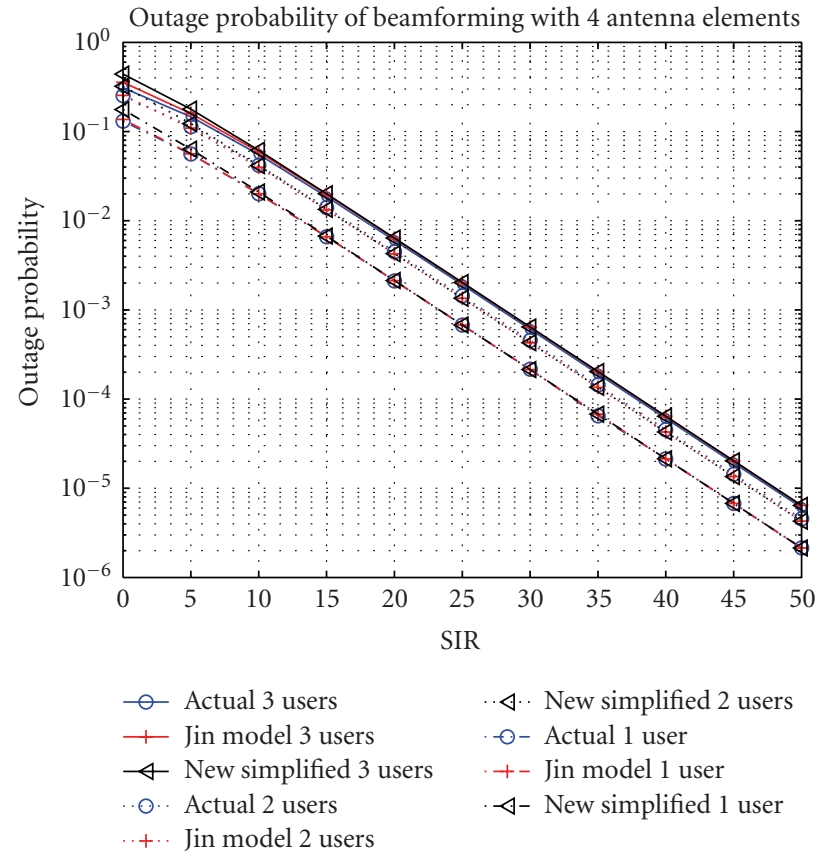

FIGURE 4: Accuracy of the new simplified beamforming model compared with Jin's model and exact result. The number of antenna elements $M=4$.

plotted. The accuracy of the simplified beamforming model can be concluded that the model becomes more accurate at higher SIR and the outage probability error introduced by the simplified beamforming model is inversely proportional to the square of average SIR. 


\section{Derivation of Outage Probabilities}

In wireless communications, adequate signal-to- interference -plus-noise ratio (SINR) is essential for successful communications $[20,21]$. Therefore, the outage probability, defined as the probability of not being able to achieve a SINR sufficient to give satisfactory reception, is an important measure in the evaluation of performance of wireless systems. Mathematically the outage probability $P_{\text {out }}$ is given by

$$
P_{\text {out }}=\int_{0}^{R} p_{\gamma}(\gamma) d \gamma=\operatorname{Pr}\left\{\frac{x}{y+n}<R\right\}
$$

in which $\gamma$ is the instantaneous $\operatorname{SINR}, p_{\gamma}(\gamma)$ is the probability density function (pdf) of $\gamma$, and $R$ is a required threshold. The variable $y$ is a function of $x, y$ and $n$, with $x$ denoting the desired signal power, $y$ denoting the total interference power and $n$ denoting the noise power. For the derivations presented here, the interferers (if any) include multiple user interference and signals of the above discussed three types (namely, barrage, partial-band or tone interferers). Without loss of generality, we can investigate the outage probability of the first user. In this section, we only consider the spectral and spatial characterization of the system and assume all the users and interferences are present in the band where the first user is active. The temporal characterization of the system and interferences is investigated in the next section.

The received signals on each antenna elements are combined by beamforming network and feed into the RAKE receiver. Assuming maximum ratio combining (MRC) technique is used, and following [22], the signal used to estimate the 0th symbol of the first user can be written as

$$
U=\sum_{n=0}^{L-1}\left[S_{n}+I_{\mathrm{mai}, n}+I_{\mathrm{si}, n}+I_{\mathrm{yi}, n}+I_{\mathrm{ni}, n}\right],
$$

where $S_{n}$ is the signal, $I_{\text {mai, } n}$ is the multiple access interference from coexisting users, $I_{\mathrm{si}, n}$ is the self interference due to multipath, $I_{\mathrm{yi}, n}$ is the jamming interference, and $I_{\mathrm{ni}, n}$ is the noise

$$
\begin{aligned}
S_{n}= & \sqrt{\frac{P}{2}} d_{0}^{1} T \sqrt{G\left(\theta_{1, n}, \theta_{1, n}\right)} \beta_{1, n}^{2}, \\
I_{\text {mai }, n}= & \sqrt{\frac{P}{2} \sum_{k=2}^{K} \sum_{l=0}^{L-1} \beta_{1, n} \beta_{k, l} \sqrt{G\left(\theta_{1, n}, \theta_{k, l}\right)}} \\
& \times\left[d_{-1}^{k} R W_{k 1}\left(\tau_{n, l}^{k}\right)+d_{0}^{k} \widehat{R W}_{k 1}\left(\tau_{n, l}^{k}\right)\right] \cos \left(\varphi_{n, l}^{k}\right), \\
I_{\mathrm{si}, n}= & \sqrt{\frac{P}{2}} \sum_{l=0, l \neq n}^{L-1} \beta_{1, n} \beta_{1, l} \sqrt{G\left(\theta_{1, n}, \theta_{1, l}\right)} \\
& \times\left[d_{-1}^{1} R W_{11}\left(\tau_{n, l}^{1}\right)+d_{0}^{1} \widehat{R W}_{11}\left(\tau_{n, l}^{1}\right)\right] \cos \left(\varphi_{n, l}^{1}\right),
\end{aligned}
$$

$$
\begin{aligned}
I_{\mathrm{yi}, n} & =\int_{n T_{c}}^{T+n T_{c}} y(t) \sqrt{G\left(\theta_{1, n}, \theta_{y}\right)} \beta_{1, n} c_{1}(t) \cos \left(\varphi_{1, n}\right) d t, \\
I_{\mathrm{ni}, n} & =\int_{n T_{c}}^{T+n T_{c}} n(t) \beta_{1, n} c_{1}(t) \cos \left(\varphi_{1, n}\right) d t,
\end{aligned}
$$

where $d_{0}^{1}$ is the information bit of the first user, $d_{-1}^{1}$ is its preceding bit, $\tau_{n, l}^{k}=\tau_{k, l}-\tau_{1, n}, \varphi_{n, l}^{k}=\varphi_{k, l}-\varphi_{1, n}, \theta_{i, j}$ is the DOA of the path $j$ of the user $i, R W$ and $\widehat{R W}$ are partial correlation function between spreading codes, where they are defined as [22]

$$
\begin{aligned}
& R W_{k 1}(\tau)=\int_{0}^{\tau} c_{k}(t-\tau) c_{1}(t) d t, \\
& \widehat{R W}_{k 1}(\tau)=\int_{\tau}^{T} c_{k}(t-\tau) c_{1}(t) d t .
\end{aligned}
$$

Performance of CDMA system has been analyzed using Standard Gaussian approximation (SGA), improved Gaussian approximation (IGA), and simplified IGA (SIGA) to model the interference statistics [23]. In this paper, we follow the analysis in [22] and use SGA to approximate the interference. The variance of the $n$th RAKE finger due to multiple access interference is give by

$$
\sigma_{\mathrm{mai}, n}^{2}=\frac{E_{b} T}{6 G_{\mathrm{DS}}} \beta_{n}^{2} \sum_{k=2}^{K} \sum_{l=0}^{L-1} \Omega_{l}^{k} G\left(\theta_{1, n}, \theta_{k, l}\right) .
$$

The variance of self interference is approximated by

$$
\sigma_{\mathrm{si}, n}^{2} \approx \frac{E_{b} T}{4 G_{\mathrm{DS}}} \beta_{n}^{2} \sum_{l=2, l \neq n}^{L-1} \Omega_{l}^{1} G\left(\theta_{1, n}, \theta_{1, l}\right)
$$

The variance of AWGN is

$$
\sigma_{\mathrm{ni}, n}^{2}=\frac{T N_{0}}{4} \beta_{n}^{2} .
$$

The impact of jamming interference is analyzed for different types of interferers.

4.1. Barrage Interference. Barrage interferers transmit bandlimited signals at high power and the performance of a spread spectrum signal is the same in the scenario of either AWGN or barrage interferers [24]. If there are $J_{1}$ barrage interferers present, and for the $j$ th interferer, denoting its average power as $Y_{1, j}$, its direction of arrival angle is $\theta_{1, j}$, and its bandwidth is $B_{1, j}$, the variance of barrage interference is given by

$$
\sigma_{J_{1}, n}^{2}=\sum_{i=1}^{J_{1}} \frac{T Y_{1, i}}{4 G_{\mathrm{DS}} B_{1, i}} \beta_{n}^{2} G\left(\theta_{1, n}, \theta_{1, i}\right)
$$

4.2. Partial-Band Interference. Partial-band interferers occupy part of the hoped bandwidth. A partial-band interferer to a DS/FH signal is the same as a partial-band jammer to a spread spectrum signal. The received power 
of the $j$ th partial-band interferer, with transmit power $y_{2, j}$, after despreading is given by [24]

$$
\tilde{y}_{2, j}=y_{2, j} \times \frac{1}{B_{2, j}} \int_{-B_{2, j}}^{B_{2, j}} \frac{\sin ^{2}\left[\left(\Delta f_{j}-f\right) 2 / B\right]}{\left[\left(\Delta f_{j}-f\right) 2 / B\right]^{2}} d f .
$$

In the above equation $B_{2, j}$ is the bandwidth of the $j$ th partial-band interferer, and $\Delta f_{j}$ is the frequency offset between the $j$ th interferer and the user. Assuming that there are $J_{2}$ partial-band interferers, each with the average transmit power of the $j$ th interferer denoted as $Y_{2, j}$, we obtain the variance of partial-band interference

$$
\sigma_{J_{2}, n}^{2}=\sum_{i=1}^{J_{2}} \frac{T Y_{2, i} \alpha_{2, j}}{4 G_{\mathrm{DS}} B} \beta_{n}^{2} G\left(\theta_{1, n}, \theta_{2, i}\right),
$$

in which $\alpha_{2, j}$ is a coefficient and it can be numerically calculated as

$$
\alpha_{2, j}=\frac{1}{B_{2, j}} \int_{-B_{2, j}}^{B_{2, j}} \frac{\sin ^{2}\left[\left(\Delta f_{j}-f\right) 2 / B\right]}{\left[\left(\Delta f_{j}-f\right) 2 / B\right]^{2}} d f .
$$

4.3. Tone Interference. The received power of a single tone interferer, with transmit power $y_{3, j}$, after despreading is found to be [24]

$$
\begin{aligned}
\tilde{y}_{3, j}= & y_{3, j} \times \frac{\sin ^{2}\left(\Delta W_{j} / 2 B\right)}{2\left(\Delta W_{j} / 2 B\right)^{2}} \\
& \times\left(1+\frac{\cos \left(2 \Delta \phi_{j}+G_{\mathrm{DS}}\left(\Delta W_{j} / B\right)\right) \sin \left(G_{\mathrm{DS}}\left(\Delta W_{j} / B\right)\right)}{G_{\mathrm{DS}} \sin \left(\Delta W_{j} / B\right)}\right),
\end{aligned}
$$

in which $\Delta W_{j}$ and $\Delta \phi_{j}$ are the frequency and phase offset between the $j$ th interferer and the signal. Assuming that there are $J_{3}$ tone interferers and the average transmit power of interferer $j$ is denoted as $Y_{3, j}$, the variance of tone interference is

$$
\sigma_{J_{3}, n}^{2}=\sum_{i=1}^{J_{3}} \frac{T Y_{3, i} \alpha_{3, j}}{4 G_{\mathrm{DS}} B} \beta_{n}^{2} G\left(\theta_{1, n}, \theta_{2, i}\right),
$$

in which $\alpha_{3, j}$ is a coefficient and

$$
\begin{aligned}
\alpha_{3, j}= & \frac{\sin ^{2}\left(\Delta W_{j} / 2 B\right)}{2\left(\Delta W_{j} / 2 B\right)^{2}} \\
& \times\left(1+\frac{\cos \left(2 \Delta \phi_{j}+G_{\mathrm{DS}}\left(\Delta W_{j} / B\right)\right) \sin \left(G_{\mathrm{DS}}\left(\Delta W_{j} / B\right)\right)}{G_{\mathrm{DS}} \sin \left(\Delta W_{j} / B\right)}\right) .
\end{aligned}
$$

4.4. Different Interference Types. Assuming that there are $J_{1}$ barrage interferers (each with average transmit power $Y_{1, j}$ ), $J_{2}$ partial-band interferers (each with average transmit power $Y_{2, j}$ ), and $J_{3}$ tone interferers (each with average transmit power $\left.Y_{3, j}\right)$ at the same time, the variance of the total interference is

$$
\sigma_{T}^{2}=\sum_{n=0}^{L-1}\left\{\sigma_{\mathrm{mai}, n}^{2}+\sigma_{\mathrm{si}, n}^{2}+\sigma_{\mathrm{ni}, n}^{2}+\sum_{i=1}^{3} \sigma_{J_{i}, n}^{2}\right\} .
$$

The desired signal is

$$
U_{S}= \pm \sqrt{\frac{E_{b} T}{2}} \sum_{n=0}^{L-1} \beta_{n}^{2} G\left(\theta_{1, n}, \theta_{1, n}\right)
$$

The SINR after maximum ratio combining is

$$
\gamma=\frac{U_{S}^{2}}{2 \sigma_{T}^{2}}=\sigma_{0} \sum_{n=0}^{L-1} \beta_{n}^{2}
$$

where

$$
\begin{aligned}
\sigma_{0}=\left[\frac{2 \sum_{k=2}^{K} \sum_{l=0}^{L-1} \Omega_{l}^{k} G\left(\theta_{1, n}, \theta_{k, l}\right)}{3 G_{\mathrm{DS}}}\right. \\
+\frac{\sum_{l=1}^{L-1} \Omega_{1}^{l} G\left(\theta_{1, n}, \theta_{1, l}\right)}{G_{\mathrm{DS}}}+\frac{n_{0}}{E_{b}} \\
\left.+\sum_{i=1}^{3} \sum_{j=1}^{J_{i}} \frac{T Y_{i, j} \alpha_{i, j}}{4 G_{\mathrm{DS}} B} \beta_{n}^{2} G\left(\theta_{1, n}, \theta_{i, j}\right)\right]^{-1} .
\end{aligned}
$$

Applying the simplified beamforming model and replace the beamforming pattern by $\alpha_{\mathrm{BF}}$, we can simplify the $\sigma_{0}$ to

$$
\begin{aligned}
\sigma_{0}\left(K, J_{1}, J_{2}, J_{3}\right)= & {\left[\frac{2(K-1) \alpha_{\mathrm{BF}}}{3 G_{\mathrm{DS}}}+\frac{\left(1-\Omega_{0}\right) \alpha_{\mathrm{BF}}}{G_{\mathrm{DS}}}\right.} \\
& \left.+\frac{1}{\Gamma_{N}}+\sum_{i=1}^{3} \sum_{j=1}^{J_{i}} \frac{\alpha_{i, j} \alpha_{\mathrm{BF}}}{G_{\mathrm{DS}} \Gamma_{i, j}}\right]^{-1},
\end{aligned}
$$

in which $\Gamma_{i, j}=X_{0} / Y_{i, j}$ is average SIR corresponding to interferer $j$ of the $i$ th type. $\alpha_{i, j}$ is its coefficient; $\alpha_{1, j}=B / B_{1, j}$, $\alpha_{2, j}$ and $\alpha_{3, j}$ can be found by (20) and (23). If the power of multiple paths is equally distributed, for example, $\delta=0$, the outage probability can be derived as

$$
P_{\text {out }}\left(K, J_{1}, J_{2}, J_{3}\right)=1-\frac{\Gamma\left(L, R /\left(\sigma_{0}\left(K, J_{1}, J_{2}, J_{3}\right) \Omega_{1}\right)\right)}{\Gamma(L)} .
$$

If the power of multiple paths is mutually different, for example, $\delta>0$, the outage probability can be given as

$P_{\text {out }}\left(K, J_{1}, J_{2}, J_{3}\right)$

$=\left[\prod_{i=0}^{L-1} \frac{1}{\sigma_{0}\left(K, J_{1}, J_{2}, J_{3}\right) \Omega_{i}}\right]$

$\times \sum_{j=0}^{L-1} \frac{e^{-R / \sigma_{0}\left(K, J_{1}, J_{2}, J_{3}\right) \Omega_{j}}}{\prod_{k=0, k \neq j}^{L-1}\left(1 /\left(\sigma_{0}\left(K, J_{1}, J_{2}, J_{3}\right) \Omega_{k}\right)-1 /\left(\sigma_{0}\left(K, J_{1}, J_{2}, J_{3}\right) \Omega_{j}\right)\right)}$. 


\section{Error Performance}

5.1. Average Outage Probability. The outage probability of a $\mathrm{DS} / \mathrm{FH}$ system is analyzed in the previous section without considering the temporal characterization of interferers and signals. The temporal characterization of an interferer can be represented by its duty cycle which is the proportion of time during which the interferer is operated. Considering that the duty cycle of an interferer is $\rho_{i, j}$, the probability that the interferer is present in a certain channel (slot) is $\rho_{i, j} / N$, in which $N$ is the total number of available channels. Let the total number of interferers of the three interference types be denoted as $J_{1}, J_{2}$, and $J_{3}$, respectively. If $K$ users are randomly hopping among the $N$ channels and all the users have the same power and all the interferers of a given type have the same transmitting power, duty cycle, and coefficients, the outage probability of the first user is obtained as

$$
P_{\text {out }}=\sum_{j_{1}=0}^{J_{1}} \sum_{j_{2}=0}^{J_{2}} \sum_{j_{3}=0}^{J_{3}} \sum_{k=0}^{K-1} P_{\text {out }}\left(k+1, j_{1}, j_{2}, j_{3}\right) p_{k} \prod_{i=1}^{3}\left(p_{j_{i}}\right),
$$

where

$$
\begin{aligned}
& p_{k}=\left(\begin{array}{c}
K^{-1} \\
k
\end{array}\right)\left(\frac{1}{N}\right)^{k}\left(1-\frac{1}{N}\right)^{(K-1-k)}, \\
& p_{j_{i}}=\left(\begin{array}{c}
J_{i} \\
J_{i}
\end{array}\right)\left(\frac{\rho_{i}}{N}\right)^{j_{i}}\left(1-\frac{\rho_{i}}{N}\right)^{\left(J_{i}-j_{i}\right)},
\end{aligned}
$$

where $P_{\text {out }}\left(k, j_{1}, j_{2}, j_{3}\right)$ can be (29) or (30) depending the value of decay factor $\delta$.

5.2. Packet Error Probability. Reed-Solomon (RS) code is an effective FEC coding scheme used in packet transmissions. If the code length is $L_{\mathrm{RS}}$ and its symbol error correction capability is $R_{C}$, the packet error probability is

$$
P_{e}=1-\sum_{i=0}^{R_{C}}\left(\begin{array}{c}
L_{\mathrm{RS}} \\
i
\end{array}\right) P_{\mathrm{out}}^{i}\left(1-P_{\mathrm{out}}\right)^{L_{\mathrm{RS}}-i}
$$

in which the outage probability $P_{\text {out }}$ can be calculated using the results in (31). The above equation is derived under the assumption that the outage probability of each symbol is independent from each other. This can be accomplished by using an interleaved RS code [25]. Such an assumption is used in this paper to focus the impact of beamforming rather than the wireless channel time coherence on the hybrid DS/FH system.

\section{Numerical Analysis}

This section presents numerical results based on the derivations in Sections 4 and 5. For the performance evaluation presented here, each user in the DS/FH spread spectrum system is considered to have a DS spreading gain $G_{\mathrm{DS}}=$ 50 and occupy a $1 \mathrm{MHz}$ bandwidth (i.e., $B=1 \mathrm{MHz}$ ) in each frequency slot, which is reflective of a transmission bandwidth of a frequency hopping signal. The propagation

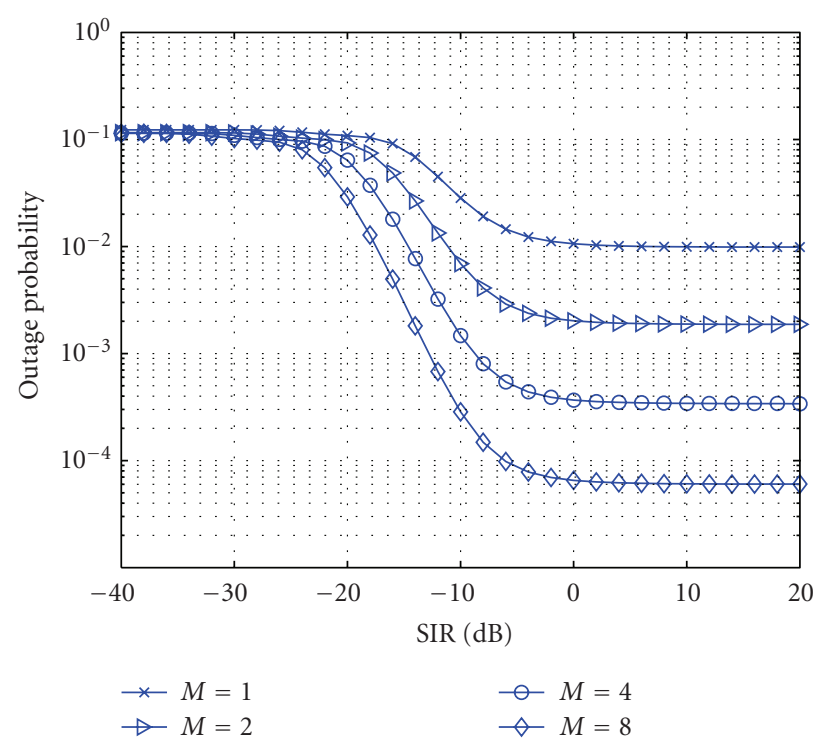

Figure 5: Outage probability of a DS/FH system, impact of beamforming, processing gain $G_{\mathrm{DS}}=50$, the number of users $K=40$, the number of interferers is $J_{1}=1, J_{2}=2, J_{3}=3$, duty cycles of interferes are $\rho_{1}=\rho_{2}=\rho_{3}=0.2$, SNR $\Gamma_{N}=100 \mathrm{~dB}$, the number of hopping channels $N=10$, the number of multiple path $L=3$, decay factor $\delta=0$, and protection ratio $r_{\text {th }}=3 \mathrm{~dB}$.

of signals from the desired transmitter as well as interfering sources to the receiver in a typical environment where such devices as those that operate in the ISM band used is well modelled through Rayleigh fading. It is reasonable to assume that the transmissions from the various sources are independent of each other. Therefore, all the signals at the receiver are considered to have undergone mutually independent Rayleigh fading.

The performance of a DS/FH system using beamforming with different number of antenna elements is plotted in Figure 5. It is seen that beamforming significantly improves the system performance under various SIR conditions. Beamforming with antenna elements of 2, 4, and 8 are compared with the case without beamforming $(M=1)$. For the three types of interferers, the duty cycle of each is 0.2 , and the numbers of interferers are 1, 2, and 3, respectively. The SNR is assumed to be $100 \mathrm{~dB}$ and spreading gain within each frequency slot is 50 . The total number of users is 40 , protection threshold equals $3 \mathrm{~dB}$, decay factor $\delta$ is 0 , and the number of frequency slots $N$ is 40. The bandwidth of the barrage interferers is assumed to be $10 \mathrm{MHz}$ which is an approximate based on the observation in Figure 2. It is noticed that about $3 \mathrm{~dB}$ gain is achieved as the number of antenna elements doubles when SIR is relatively low (SIR is around $-20 \mathrm{~dB}$ ). When multiuser interference dominates the system performance (at high SIR), increasing the number of antenna elements also reduces the outage probability significantly. This illustrates that beamforming is an effective technique which reduces interference due to either coexisting DS/FH signals or other interferers. 


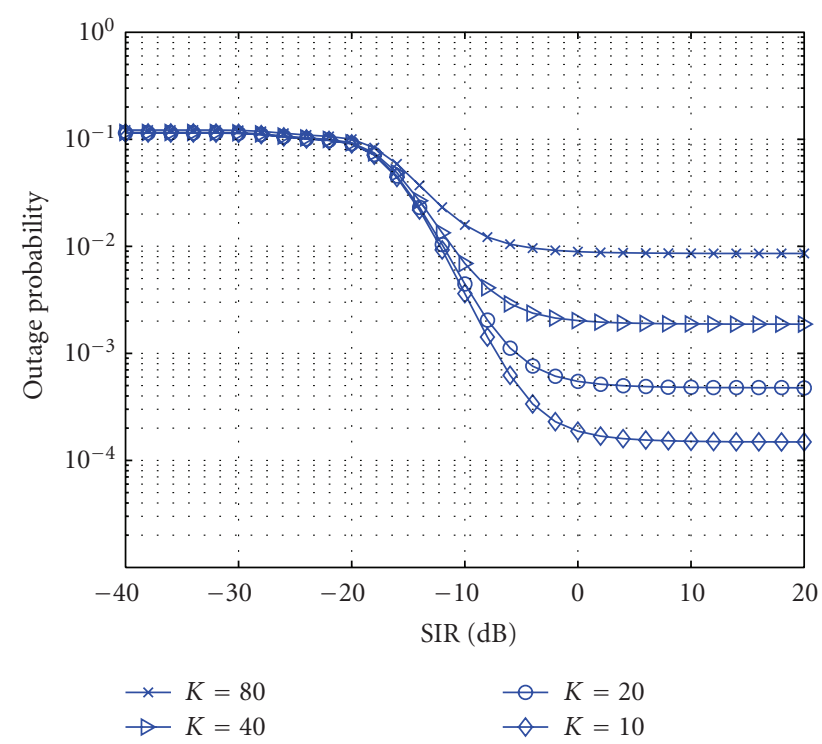

Figure 6: Outage probability of a DS/FH system; Impact of the number of users, the number of antenna elements $M=2$, processing gain $G_{\mathrm{DS}}=50$, the number of interferers is $J_{1}=1, J_{2}=$ $2, J_{3}=3$, duty cycles of interferes are $\rho_{1}=\rho_{2}=\rho_{3}=0.2$, SNR $\Gamma_{N}=100 \mathrm{~dB}$, the number of hopping channels $N=10$, the number of multiple path $L=3$, decay factor $\delta=0$, and protection ratio $r_{\text {th }}=3 \mathrm{~dB}$.

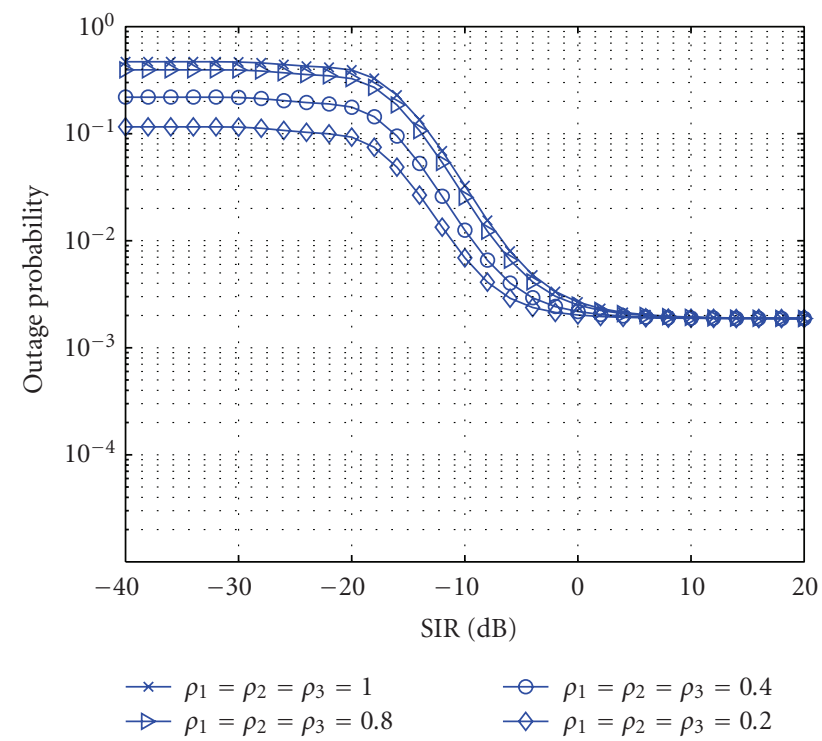

FIGURE 7: Outage probability of a DS/FH system, impact of the duty cycle of interferers, the number of antenna elements $M=2$, processing gain $G_{\mathrm{DS}}=50$, the number of users $K=40$, the number of interferers is $J_{1}=1, J_{2}=2, J_{3}=3$, SNR $\Gamma_{N}=100 \mathrm{~dB}$, the number of hopping channels $N=10$, the number of multiple path $L=3$, decay factor $\delta=0$, and protection ratio $r_{\text {th }}=3 \mathrm{~dB}$.

The impact of multiple users in the system is shown in Figure 6. Outage probability results of a system with 10, 20,40 , and 80 users are compared. The number of antenna elements is assumed to be 2 and other parameters are the

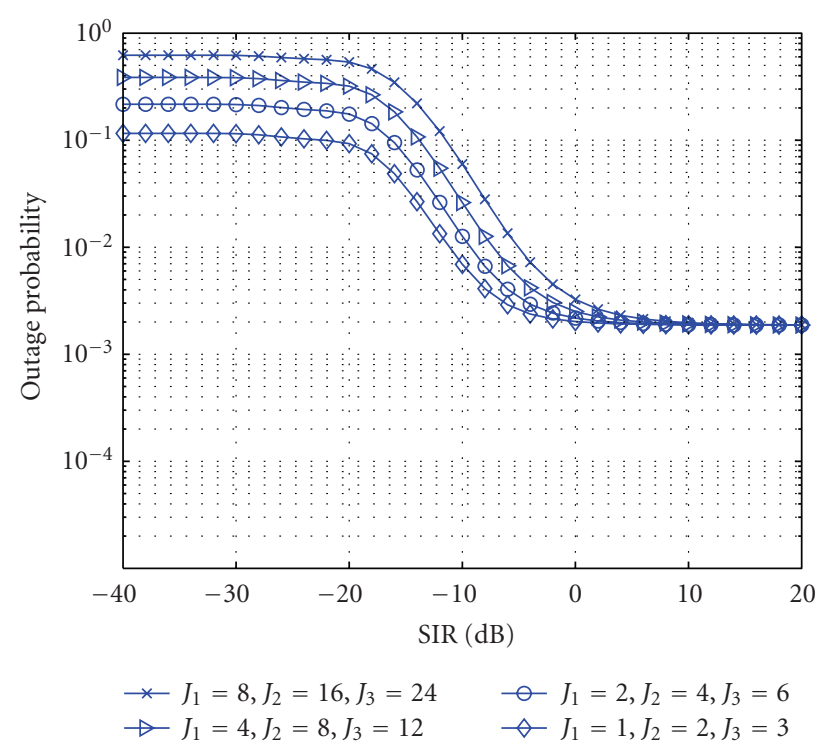

FIgURE 8: Outage probability of a DS/FH system; Impact of the number of interferers, the number of antenna elements $M=2$, processing gain $G_{D S}=50$, the number of users $K=40$, duty cycles of interferes are $\rho_{1}=\rho_{2}=\rho_{3}=0.2$, SNR $\Gamma_{N}=100 \mathrm{~dB}$, the number of hopping channels $N=10$, the number of multiple path $L=3$, decay factor $\delta=0$, and protection ratio $r_{\text {th }}=3 \mathrm{~dB}$.

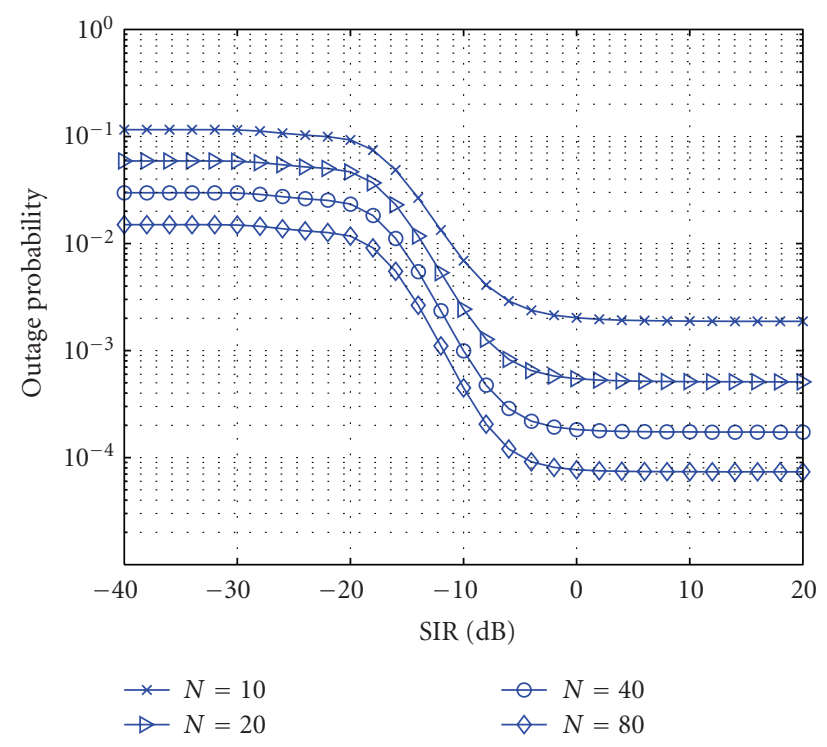

FIGURE 9: Outage probability of a DS/FH system, impact of the number of hopping channels, the number of antenna elements $M=2$, processing gain $G_{D S}=50$, the number of users $K=40$, the number of interferers is $J_{1}=1, J_{2}=2, J_{3}=3$, duty cycles of interferes are $\rho_{1}=\rho_{2}=\rho_{3}=0.2$, SNR $\Gamma_{N}=100 \mathrm{~dB}$, the number of multiple path $L=3$, decay factor $\delta=0$, and protection ratio $r_{\text {th }}=3 \mathrm{~dB}$.

same as those in Figure 5. It is seen that if multiple users are present in the system, increasing SIR does not always decrease the outage probability. This is due to the fact that multiple user interference in the DS/FH system will dominate the system performance as SIR increases. 


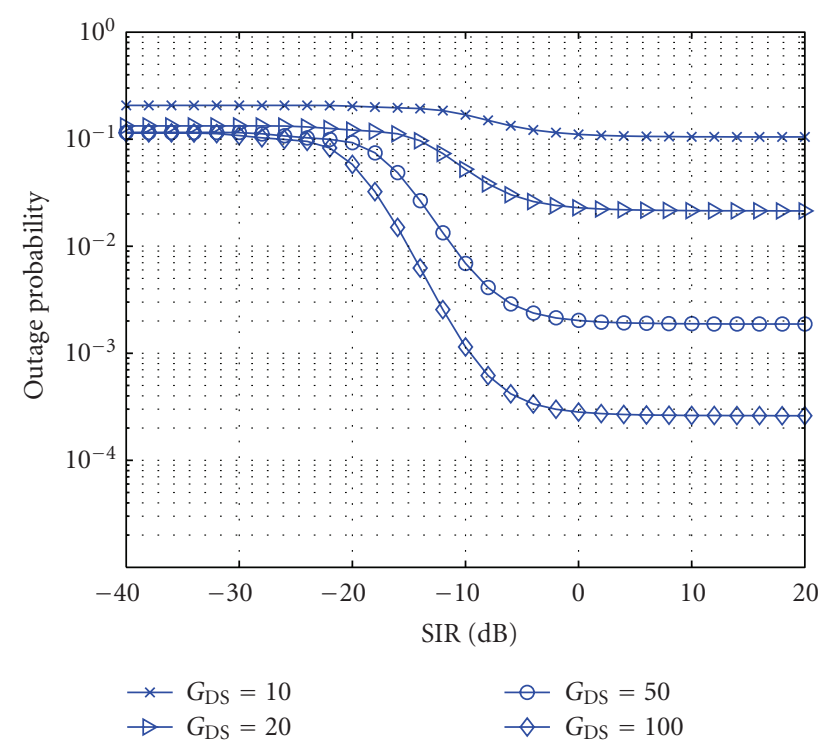

Figure 10: Outage probability of a DS/FH system, impact of processing gain, the number of antenna elements $M=2$, the number of users $K=40$, the number interferers are $J_{1}=1, J_{2}=$ 2 , $J_{3}=3$, duty cycles of interferes are $\rho_{1}=\rho_{2}=\rho_{3}=0.2$, SNR $\Gamma_{N}=100 \mathrm{~dB}$, the number of hopping channels $N=10$, the number of multiple path $L=3$, decay factor $\delta=0$, and protection ratio $r_{\text {th }}=3 \mathrm{~dB}$.

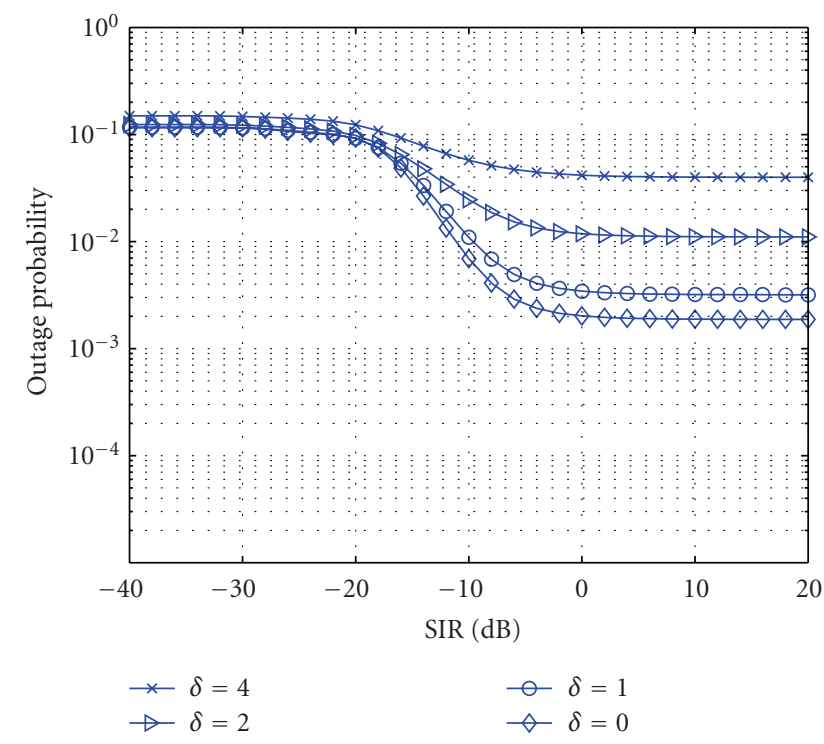

FIGURE 11: Outage probability of a DS/FH system, impact of the decay factor, the number of antenna elements $M=2$, processing gain $G_{\mathrm{DS}}=50$, the number of users $K=40$, the number interferers are $J_{1}=1, J_{2}=2, J_{3}=3$, duty cycles of interferes are $\rho_{1}=\rho_{2}=$ $\rho_{3}=0.2$, SNR $\Gamma_{N}=100 \mathrm{~dB}$, the number of hopping channels $N=$ 10 , the number of multiple path $L=3$, and protection ratio $r_{\text {th }}=$ $3 \mathrm{~dB}$.

The impacts of ISM band interference duty cycles, the number of interferers, and the number of frequency slots are examined in Figures 7, 8, and 9, respectively. It is seen

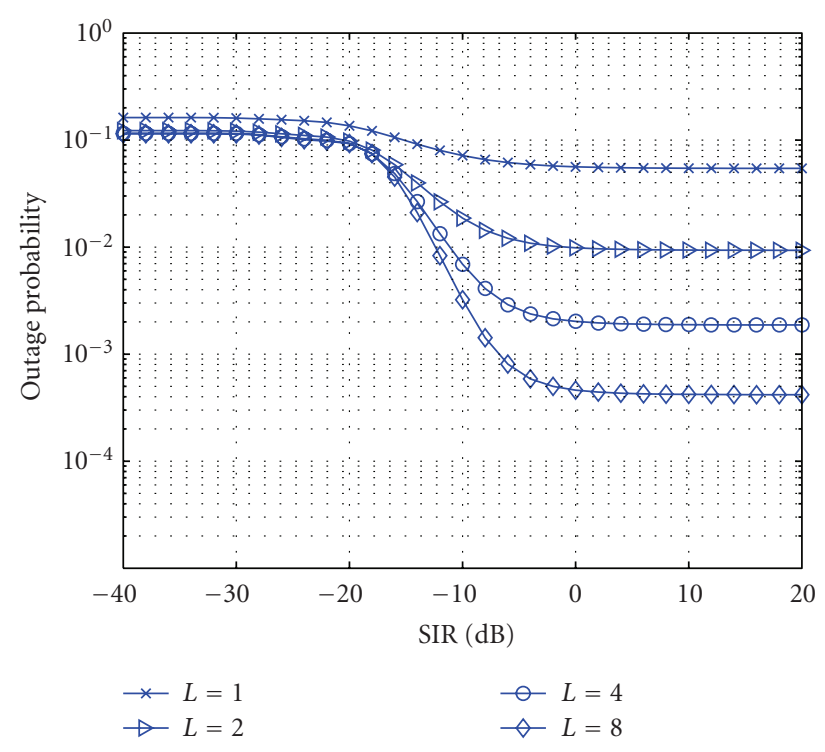

FIGURE 12: Outage probability of a DS/FH system, impact of the number of multipath, the number of antenna elements $M=2$, processing gain $G_{\mathrm{DS}}=50$, the number of users $K=40$, the number of interferers is $J_{1}=1, J_{2}=2, J_{3}=3$, duty cycles of interferes are $\rho_{1}=\rho_{2}=\rho_{3}=0.2$, SNR $\Gamma_{N}=100 \mathrm{~dB}$, the number of hopping channels $N=10$, decay factor $\delta=0$, and protection ratio $r_{\text {th }}=3 \mathrm{~dB}$.

that the duty cycles and the number of interferers have greater impact on the outage probability at lower SIR, where ISM band interference dominates the system as compared to DS/FH multiple access interference. Those impacts diminish at higher SIR when multiuser interference becomes the major concern in the system. It is also seen that increasing the number of frequency slots improves the system performance regardless of the signal to interference ratio. This is due to the increase of the processing gain of the DS/FH system.

The impact of spreading gain is shown in Figure 10. It is seen that increasing the spreading gain improves the outage performance at all SIR ranges. This is due to the fact that increasing the spreading gain can reduce multiple access interference at lower SIR and decrease the self-interference at higher SIR.

The impact of fading channel is shown in Figures 11 and 12. It is seen in Figure 11 that the outage performance becomes worse if the signal strength on multiple paths decays fast. Figure 12 illustrates that the more equally distributed paths are in the fading channel the better outage performance the system has. Those performance improvements can be explained by more effective diversity in the fading channel.

\section{Conclusion}

In this paper, we have investigated the issue of coexistence interference in unlicensed bands. Motivation for the work originates from the widespread use of the $2.4 \mathrm{GHz}$ ISM band for varied services and the growing realization of the inadequacy of the licensing methodology for spectrum 
management that is currently in place. The paradigm for operation in an unlicensed band is to minimize the interference caused to other users in the band and to avoid and suppress the interference from coexisting devices. Based on our measurement studies in the $2.4 \mathrm{GHz}$ ISM band, an analytical interference model has been developed in this paper. The modeling approach adopted involves profiling the observed emissions under different interference types based on their spectral characteristics. The model also considers the temporal characterizations and power levels of all the emissions. The effect of coexistence interference in the $2.4 \mathrm{GHz}$ ISM band on the performance of a hybrid DS/FH system using beamforming, in terms of outage probability and packet error probability, has also been evaluated in this paper. Results of numerical evaluation of the derived performance measures have been presented. This study is part of our ongoing effort to develop an analytical framework for interference modeling and performance evaluation in the unlicensed band, as it is seen as an invaluable tool for dynamic spectrum access system designs.

\section{Acknowledgments}

The authors would like to acknowledge and thank Mr. Jason Evans, Dr. Ufuk Tureli, Dr. Paul Kolodzy, and Dr. Joseph Evans for their support and contributions.

\section{References}

[1] "Code of federal regulations (CFR) title 47: telecommunication-part 15: radio frequency devices," October 2004, http://www.access.gpo.gov/nara/cfr/waisidx_04/ 47cfr15_04.html.

[2] P. B. Kenington and D. W. Bennett, "Field measurements study into the potential effects of ISM emissions on cellular radio equipment," IEE Proceedings: Science, Measurement and Technology, vol. 144, no. 3, pp. 134-140, 1997.

[3] C. C. Huang and R. Khayata, "Delay spreads and channel dynamics measurements at ISM bands," in Proceedings of IEEE International Conference on Communications-Discovering a New World of Communications (SUPERCOMM/ICC '92), vol. 3, pp. 1222-1226, Chicago, Ill, USA, June 1992.

[4] K. Giannopoulou, A. Katsareli, D. Dres, D. Vouyioukas, and P. Constantinou, "Measurements for $2.4 \mathrm{GHz}$ spread spectrum system in modern office buildings," in Proceedings of the 10th Mediterranean Electrotechnical Conference (MELECON '00), vol. 1, pp. 326-329, May 2000.

[5] M. Biggs, A. Henley, and T. Clarkson, "Occupancy analysis of the $2.4 \mathrm{GHz}$ ISM band," IEE Proceedings: Communications, vol. 151, no. 5, pp. 481-488, 2004.

[6] T. Kamakaris and J. Evans, "Distributed spectrum measurements," in Proceedings of the 24th Annual Joint Conference of the IEEE Computer and Communications Societies (INFOCOM '05), March 2005.

[7] H. Li, M. Syed, and Y.-D. Yao, "Outage performance of a hybrid DS/FH spread-spectrum signal in an ISM band," International Journal of Network Management, vol. 16, no. 2, pp. 115-129, 2006.

[8] H. L. V. Trees, Optimum Array Processing, Part IV of Detection, Estimation, and Modulation Theory, Wiley-Interscience, New York, NY, USA, 2002.
[9] “MSS Comblock Modules," http://www.comblock.com.

[10] "The bluetooth special interest group," May 2009, http://www.bluetooth.com.

[11] IEEE Standard 802.11b-1999 (Supplement to ANSI/IEEE Standard 802.11, 1999 Edition), 1999.

[12] A. Hoshikuki, M. Yamamoto, S. Ishii, R. Kohno, and H. Imai, "Implementation of an industrial R/C system using a hybrid DS/FH spread spectrum technique," in Proceedings of the 2nd IEEE International Symposium on Spread Spectrum Techniques and Applications, pp. 179-182, November-December 1992.

[13] P. Varzakas and G. S. Tombras, "Spectral efficiency for a hybrid DS/FH code-division multiple-access system in cellular mobile radio," IEEE Transactions on Vehicular Technology, vol. 50, no. 6, pp. 1321-1327, 2001.

[14] J. G. Proakis, Digital Communications, McGraw-Hill, New York, NY, USA, 3rd edition, 1995.

[15] S. Haykin, Adaptive Filter Theory, Prentice-Hall, Upper Saddle River, NJ, USA, 3rd edition, 1986.

[16] A. F. Naguib, A. Paulraj, and T. Kailath, "Capacity improvement with base-station antenna arrays in cellular CDMA," IEEE Transactions on Vehicular Technology, vol. 43, no. 3, pp. 691-698, 1994.

[17] U. Spagnolini, "A simplified model for probability of error in DS CDMA systems with adaptive antenna arrays," in Proceedings of IEEE International Conference on Communications (ICC '01), vol. 7, pp. 2271-2275, June 2001.

[18] J. Yu and Y.-D. Yao, "Reverse link capacity of SIR-based powercontrolled CDMA systems with antenna arrays," Wireless Communications and Mobile Computing, vol. 3, no. 6, pp. 759$772,2003$.

[19] H. Li, Y.-D. Yao, and J. Yu, "Outage probabilities of wireless systems with imperfect beamforming," IEEE Transactions on Vehicular Technology, vol. 55, no. 5, pp. 1503-1515, 2006.

[20] Y.-D. Yao and A. U. H. Sheikh, "Investigations into cochannel interference in microcellular mobile radio systems," IEEE Transactions on Vehicular Technology, vol. 41, no. 2, pp. 114$123,1992$.

[21] Y.-D. Yao and A. U. H. Sheikh, "Outage probability analysis for microcell mobile radio systems with cochannel interferers n Rician/Rayleigh fading environment," Electronics Letters, vol. 26, no. 13, pp. 864-866, 1990.

[22] G. P. Efthymoglou, T. Piboongungon, and V. A. Aalo, "Performance of DS-CDMA receivers with MRC in nakagami$\mathrm{m}$ fading channels with arbitrary fading parameters," IEEE Transactions on Vehicular Technology, vol. 55, no. 1, pp. 104114, 2006.

[23] J. M. Holtzman, "A simple, accurate method to calculate spread-spectrum multiple-access error probabilities," IEEE Transactions on Communications, vol. 40, no. 3, pp. 461-464, 1992.

[24] R. L. Peterson, R. E. Ziemer, and D. E. Borth, Introduction to Spread Spectrum Communications, Prentice-Hall, Upper Saddle River, NJ, USA, 1995.

[25] C. Yi and J. H. Lee, "Hybrid ARQ scheme using interleaved reed-solomon codes in a power-controlled DS-CDMA cellular system," IEEE Transactions on Vehicular Technology, vol. 45, no. 4, pp. 683-687, 1996. 